\title{
Patch testing in a case of eslicarbazepine and carbamazepine induced cutaneous reaction
}

\author{
${ }^{1}$ Immunoallergology Department, Dona Estefânia Hospital, Centro Hospitalar Lisboa Central, Lisbon, Portugal \\ ${ }^{2}$ Occupational Health Department, São José Hospital, Centro Hospitalar Lisboa Central, Lisbon, Portugal
}

\section{KEYwORDS}

eslicarbazepine; anticonvulsant drugs;

skin rash; drug allergy; epicutaneous tests

\author{
Corresponding author \\ Elena Finelli \\ Immunoallergology Department \\ Dona Estefânia Hospital \\ R. Jacinta Marto, 1169-045, Lisbon, Portugal \\ Phone: +351213126600 \\ Mobile: +351919106256 \\ E-mail: finelli.elena@gmail.com
}

Doi

10.23822/EurAnnACI.1764-1489.37

\begin{abstract}
Summary
Anticonvulsants are among the drugs most commonly involved in cutaneous adverse drug reactions (CADRs). Eslicarbazepine is a new anti-epileptic drug, chemically related to carbamazepine but with a more favorable safety profile. We report the clinical case of a woman who developed a skin rash on day 10 of eslicarbazepine with further exacerbation with eosinophilia on day 2 of carbamazepine. Epicutaneous tests were positive with eslicarbazepine.
\end{abstract}

\section{Introduction}

Eslicarbazepine is an oral anticonvulsant indicated for the adjunctive treatment of partial seizures, with or without secondary generalization, in the adult population. Eslicarbazepine acetate is a prodrug for eslicarbazepine (S-licarbazepine), which is the active metabolite of oxcarbazepine $(1,2)$. It is contraindicated in patients with a history of hypersensitivity to other carboxamide derivatives, such as oxcarbazepine and carbamazepine, since it shares a similar chemical structure (3). Eslicarbazepine shows a better safety profile than carbamazepine $(2,4)$; in clinical trials in adult epileptic patients, rash was reported in $1-3 \%$ of the patients taking eslicarbazepine acetate, while carbamazepine is known to cause cutaneous adverse drug reactions (cADRs) in up to $17 \%$ of patients $(2,5,6)$.

The occurrence of cADRs is a significant problem, because it often leads to discontinuation of treatment and is usually unpre- dictable. Nevertheless, recent studies have revealed that human leukocyte antigen (HLA) genotypes could be possibly linked to a predisposition to cADRs induced by drugs, including carbamazepine $(5,7)$. Since the approval of eslicarbazepine, in 2009, there have been scarce reports of hypersensitivity reactions and, currently, there are neither established risk factors for adverse reaction with eslicarbazepine acetate, nor a known correlation with the HLA genotype, as there is for carbamazepine $(4,7)$.

We report a case of a patient with adverse skin reactions to eslicarbazepine and carbamazepine, whose skin patch tests were positive for the former compound.

\section{Case Report}

A 54-year-old woman, Caucasian, developed a slightly pruritic maculo-papular generalized skin eruption without fever or systemic symptoms on the 10th day of eslicarbazepine acetate and 


\section{Figure 1}

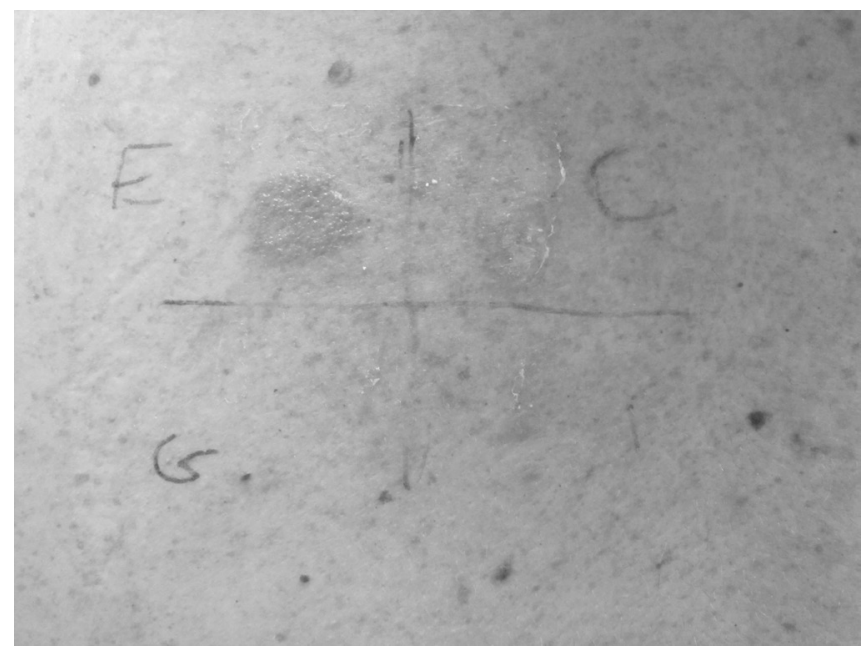

gabapentine, prescribed for a trigeminal neuropathic pain. The patient was initially medicated with $25 \mathrm{mg}$ of hidroxizine per day, and was told to maintain the previous medication. There was some improvement, but the skin rash persisted. Eleven days after the beginning of the skin eruption she was observed by her neurologist, who stopped gabapentine and eslicarbazepine and switched her to pregabalin.

After two days, due to worsening of the pain on her new therapeutic regime, the patient went to the emergency room where she was advised to stop pregabalin and started treatment with carbamazepine, metamizol and diazepam. Two days later she recurred again to the emergency because of marked worsening of the skin rash. She had a disseminated erithemato-violaceous maculo-papular eruption, along with mild edema of the face and extremities, without arthralgia or fever. Her blood tests revealed eosinophilia (14.12\%; $0.8 \times 109 / \mathrm{L})$; the serum levels of creatinine, blood urea nitrogen and hepatic parameters were within normal ranges. The patient started immediately a prednisolone course, withdrawing all previous medication, and was referred to our Immunoallergology outpatient clinic. There was a progressive regression of the skin lesions, with a complete recovery within around six weeks and normalization of the eosinophil count $(0.140 \times 109 / \mathrm{L}, 2.5 \%)$ in blood tests performed five months later. She meanwhile started treatment with amitriptyline, which was well tolerated. The previous use of antiepileptic drugs was denied.

One month after full recovery we performed patch tests with eslicarbazepine, gabapentin, carbamazepine and pregabalin. We used commercial tablets of $800 \mathrm{mg}$ eslicarbazepine, $100 \mathrm{mg}$ gabapentin, $200 \mathrm{mg}$ carbamazepine and $25 \mathrm{mg}$ pregabalin, which were finely grounded and incorporated at $20 \%$ in white petrolatum. The tests were positive only for eslicarbazepine, both at day 2 and day 4 (figure 1). Six controls were patch-tested with eslicarbazepine, in the same concentration, with negative result.

\section{Discussion}

This patient suffered from a generalized maculopapular eruption with mild eosinophilia but without systemic symptoms or evidence of organic lesion, thus not fulfilling the criteria for DRESS (8). However, antiepileptic drugs, in particular carbamazepine, are among the most common causes of severe cutaneous drug reactions $(3,5,8)$. The prompt interruption of the suspected drugs might have protected the patient from a more severe reaction $(3,5,9)$.

Given the clinical history, with the first symptoms appearing 10 days after starting eslicarbazepine and gabapentine followed by exacerbation 2 days after starting carbamazepine, which is chemically related to eslicarbazepine, we assumed eslicarbazepine and carbamazepine as the most probable culprit drugs. The positive patch test with eslicarbazepine strengthened our diagnosis. Avoidance of these and related drugs, like oxcarbazepine, was advised. Carbamazepine is among the drugs most often involved in cutaneous adverse reactions, and there are many reports about the usefulness of patch test for the diagnosis $(5,9,10)$. Conversely, eslicarbazepine is rarely implicated in hypersensitivity reactions $(2,4)$ and we found no published information concerning the use of epicutaneous testing or the determination of non irritant concentrations with this drug.

Based on the published guidelines and because we used the commercial form of the implicated drugs, we decided to test with a concentration of $20 \%$ in petrolatum $(11,12)$. The negative result on six healthy controls allowed us to exclude an irritant reaction.

Despite the negative epicutaneous test with carbamazepine, we still consider the patient might be sensitized to this drug. There are reports of negative patch tests with carbamazepine, possibly because in some cases the reaction could be triggered by a metabolite and not by the parent compound $(13,14)$. Moreover, drug hypersensitivity reactions tend to occur earlier and be more severe on reexposure, which was evident in our patient after she started carbamazepine. Alternatively, she could have suffered a flare-up reaction and not a cross-reactive hypersensitivity reaction to carbamazepine (15).

In conclusion, we consider this case relevant because there is a scarcity of reports on hypersensitivity reactions to eslicarbazepine. Epicutaneous tests with this drug seem to be useful for the identification of the culprit drug on cADRs. The concentration of $20 \%$ on petrolatum (with grounded tablets) elicited no irritant reactions.

\section{Conflict of Interest}

The authors declare that they have no conflict of interest. 


\section{References}

1. Almeida L, Soares-da-Silva P. Eslicarbazepine acetate (BIA 2-093). Neurotherapeutics 2007; 4:88-96.

2. Ben-Menachem E. Eslicarbazepine acetate: a well-kept secret? Epilepsy Currents 2010; 10(1):7-8.

3. Blaszczyk B, Lason W, Czuczwar SJ. Antiepileptic drugs and adverse skin reactions: An update. Pharmacol Rep 2015; 67:426-34.

4. Gierbolini J, Giarratano M; Benbadis SR. Carbamazepine-related antiepileptic drugs for the treatment of epilepsy - a comparative review. Expert Opin Pharmacother 2016; 17(7):885-8.

5. Ye YM, Thong BYH, Park HS. Hypersensitivity to antiepileptic drugs. Immunol Allergy Clin N Am 2014; 34:633-43.

6. Massot A, Gimenez-Arnau A. Cutaneous adverse drug reaction type erythema multiforme major induced by eslicarbazepine. J Pharmacol Pharmacother 2014; 5(4):271-4.

7. Kaniwa N, Saito Y. The risk of cutaneous adverse reactions among patients with the HLA-A* 31:01 allele who are given carbamazepine, oxcarbazepine or eslicarbazepine: a perspective review. Ther Adv Drug Saf 2013; 4(6);246-53.

8. Husain Z, Reddy BY, Schwartz RA. DRESS syndrome: Part I. Clinical perspectives. J Am Acad Dermatol 2013; 68(5):693e1-14.
9. Husain Z, Reddy BY, Schwartz RA. DRESS syndrome: Part II. Management and therapeutics. Am Acad Dermatol 2013; 68(5):709e1-9.

10. Buyuktiryaki AB, Bezirganoglu H, Sahiner UM, Yavuz ST, Tuncer A, Kara A, Sackesen C. Patch testing is an effective method for the diagnosis of carbamazepine-induced drug reaction, eosinophilia and systemic symptoms (DRESS) syndrome in an 8-year-old girl. Australas J Dermatol 2012; 53:274-7.

11. Aquino MR, Sher J, Fonacier L. Patch testing for drugs. Dermatitis 2013; 24(5):205-14.

12. Barbaud A, Gonçalo M, Buynzeel D, Bircher A. Guidelines for performing skin testsin the investigation of cutaneous adverse drug reactions. Contact Dermatitis 2001; 45:321-8.

13. Elzagallaai AA, Knowles SR, Rieder MJ, Bend JR, Shear NH, Koren G. Patch testing for the diagnosis of anticonvulsant hypersensitivity syndrome - a systematic review. Drug Safety 2009; 32(5):391-408.

14. Lee AY, Choi J, Chey WY. Patch testing with carbamazepine and its main metabolite carbamazepine epoxide in cutaneous adverse drug reactions to carbamazepine. Contact Dermatitis 2003; 48:137-9.

15. Pichler WJ, Daubner B, Kawabata T. Drug hypersensitivity: flareup reactions, cross-reactivity and multiple drug hypersensitivity. J Dermatol 2011; 38:216-21 